\title{
Partition, Reaction and Diffusion Coefficients of Bromine in Elastomeric
}

\author{
Polydimethylsiloxane - Supplementary
}

\section{Information}

Maria Eleni Moustaka, ${ }^{\dagger}$ Michael M. Norton, ${ }^{\dagger}$ Viktor Horvath, ${ }^{\dagger}$ Baptiste Blanc, ${ }^{\dagger}$ S.Ali Aghvami, ${ }^{\dagger}$ and Seth Fraden*, ${ }^{*}$

E-mail: fraden@brandeis.edu

\section{Materials}

\section{S1. Neutralizing solution}

A sodium thiosulfate neutralizing solution, supplied by Sigma Aldrich (250 g), was created prior to every experiment. We diluted $120 \mathrm{ml}$ of $\mathrm{H}_{2} \mathrm{O}$ with $30 \mathrm{~g}$ of sodium thiosulfate. This solution was used as a safety precaution while working with bromine water and for safely disposing the remaining reacted PDMS pieces and bromine water after each experiment. ${ }^{1}$ Both bromine water and PDMS pieces turned from brown-red to colorless when added to the neutralizing solution. Transparency of the PDMS pieces was not restored with this treatment. 


\section{S2. PDMS films fabrication}

The PDMS elastomer used in this study was Sylgard 184, which is supplied as two fluids that were combined at a ratio of 10:1 as suggested by the manufacturer. The components were mixed using a Thinky planetary non-vacuum centrifugal mixer AR-250 for 6 minutes. A precise amount of the degassed mixture was poured onto the center of a 3 inch diameter stainless steel wafer (0.0293 inches thickness) supplied by Stainless supply. See Material Section for more details. The wafers were then placed into a vacuum chamber and degassed for 3 minutes. The PDMS layers thickness was regulated by spin coating the stainless steel wafer using specific spinning programs at room temperature. The mass of the deposited degassed PDMS mixture was also recorded as is a significant parameter for the resulting thickness. The spin-coater used is Headway Research, Inc, model PWM32. We fabricated and used PDMS films with thicknesses $1=68 \pm 7 \mu \mathrm{m}, \mathrm{h} 2=160 \pm 15 \mu \mathrm{m}, \mathrm{h} 3=320 \pm 10 \mu \mathrm{m}$, $\mathrm{h} 4=390 \pm 20 \mu \mathrm{m}, \mathrm{h} 5=480 \pm 13 \mu \mathrm{m}$ and $\mathrm{h} 6=720 \pm 18 \mu \mathrm{m}$. Their spinning programs are described in Table S1, Table S2, Table S3, Table S4, Table S5 and Table S6 respectively. The spin-coated cross-linked PDMS layers deposited on the stainless steel wafers for all the above thicknesses were cured at $70{ }^{\circ} \mathrm{C}$ for 12 hours. The thicknesses of the PDMS layers were measured with the use of a 3D ZETA 20 Optical profilometer. The PDMS cubes with 10:1 mixing ratio shown in Fig. S3 and Fig. S6 were cast inside plastic rectangular cuvettes with side dimensions $1 \mathrm{~cm} \mathrm{x} 1 \mathrm{~cm} \mathrm{x} 3 \mathrm{~cm}$ (lwh), then were cut into $1 \mathrm{~cm} \mathrm{x} 1 \mathrm{~cm} \mathrm{x} 1 \mathrm{~cm}$ (lwh) cubes.

Table S1: Spinning program for thin PDMS films with thickness 68 um

\begin{tabular}{lll}
\hline PDMS layer Thickness: & $68 \pm 7 \mathrm{\mu m}$ & \\
Mass of PDMS mixture deposited: & $2 \mathrm{~g}$ & \\
\hline \hline Spin-coat program & Step 1 & Step 2 \\
\hline Speed (rpm) & 500 & 700 \\
Ramp (rpm/s) & 100 & 300 \\
Time (s) & 5 & 60 \\
\hline
\end{tabular}


Table S2: Spinning program for thin PDMS films with thickness $160 \mu \mathrm{m}$

\begin{tabular}{lll}
\hline PDMS layer Thickness: & $160 \pm 15 \mu \mathrm{m}$ \\
Mass of PDMS mixture deposited: & $3 \mathrm{~g}$ & \\
\hline \hline Spin-coat program & Step 1 & Step 2 \\
\hline Speed (rpm) & 500 & 500 \\
Ramp (rpm/s) & 100 & 100 \\
Time (s) & 5 & 30 \\
\hline
\end{tabular}

Table S3: Spinning program for thin PDMS films with thickness $320 \mu \mathrm{m}$

\begin{tabular}{ll}
\hline \hline PDMS layer Thickness: & $320 \pm 10 \mu \mathrm{m}$ \\
Mass of PDMS mixture deposited: & $2 \mathrm{~g}$ \\
\hline \hline Spin-coat program & Step 1 \\
\hline Speed $(\mathrm{rpm})$ & 100 \\
Ramp $(\mathrm{rpm} / \mathrm{s})$ & 100 \\
Time $(\mathrm{s})$ & 80 \\
\hline
\end{tabular}

Table S4: Spinning program for thin PDMS films with thickness $390 \mu \mathrm{m}$.

\begin{tabular}{ll}
\hline PDMS layer Thickness: & $390 \pm 20 \mu \mathrm{m}$ \\
Mass of PDMS mixture deposited: & $3 \mathrm{~g}$ \\
\hline \hline Spin-coat program & Step 1 \\
\hline Speed $(\mathrm{rpm})$ & 140 \\
Ramp $(\mathrm{rpm} / \mathrm{s})$ & 100 \\
Time $(\mathrm{s})$ & 50 \\
\hline
\end{tabular}

Table S5: Spinning program for thin PDMS films with thickness $480 \mu \mathrm{m}$

\begin{tabular}{ll}
\hline PDMS layer Thickness: & $480 \pm 13 \mu \mathrm{m}$ \\
Mass of PDMS mixture deposited: & $4 \mathrm{~g}$ \\
\hline \hline Spin-coat program & Step 1 \\
\hline Speed $(\mathrm{rpm})$ & 140 \\
Ramp $(\mathrm{rpm} / \mathrm{s})$ & 100 \\
Time $(\mathrm{s})$ & 30 \\
\hline
\end{tabular}

Table S6: Spinning program for thin PDMS films with thickness $720 \mu \mathrm{m}$

\begin{tabular}{ll}
\hline PDMS layer Thickness: & $720 \pm 18 \mu \mathrm{m}$ \\
Mass of PDMS mixture deposited: & $4 \mathrm{~g}$ \\
\hline \hline Spin-coat program & Step 1 \\
\hline Speed (rpm) & 100 \\
Ramp (rpm/s) & 100 \\
Time (s) & 10 \\
\hline
\end{tabular}




\section{S3. PDMS Density}

The estimation of the partition coefficient requires knowing the total volume of the PDMS. We weighed the PDMS and calculated the total volume using the density. According to ChaoMing Chen et. al. ${ }^{2}$ the density of PDMS decreases linearly with mixing ratio of PDMS resin to curing agent and the density value for mixing ratio 10:1 is reported to be $\rho=1.065 \mathrm{~g} / \mathrm{cm}^{3}$. By measuring the mass and the dimensions of 18 PDMS cubes $1 \mathrm{~cm} \mathrm{x} 1 \mathrm{~cm}$ x $1 \mathrm{~cm}$ (lwh), with mixing ratio of PDMS resin to curing agent 10:1 we found the PDMS density to be $\rho=$ $1.07 \pm 0.03 \mathrm{~g} / \mathrm{cm}^{3}$, which agrees with literature. ${ }^{2}$ This density value is used as a constant parameter to our fitting models.

\section{S4. Absorption spectrum and Extinction coefficient of bromine water}

We observe the wavelength of the maximum absorbance of brominated water to be $\lambda=$ $404 \mathrm{~nm}$. (see Fig. S1A), which is within the expected peak wavelengths $\lambda=392-405 \mathrm{~nm}^{3-5}$ The absorption peak in the visible spectrum of bromine gas is reported to be $\lambda=415 \mathrm{~nm}$ with an extinction coefficient of $\epsilon_{415 \mathrm{~nm}}=152 \mathrm{M}^{-1} \mathrm{~cm}^{-1} \cdot{ }^{6,7}$ When bromine is mixed with water, the absorption spectrum broadens and blue-shifts. ${ }^{3-5}$

We measured that the brominated water absorbance changes linearly as concentration of bromine increases from $2 \mathrm{mM}$ to $16 \mathrm{mM}$ at $\lambda=404 \mathrm{~nm}$. Hence, Beer's law holds for bromine concentrations lower than $16 \mathrm{mM}$ (see Fig. S1B). By fitting a line to these measurements, we determined an extinction coefficient of $\epsilon_{404 \mathrm{~nm}}=114 \pm 4 \mathrm{M}^{-1} \mathrm{~cm}^{-1}$. The only extinction coefficient that we could find in the literature for $\lambda=404 \mathrm{~nm}$ is $\epsilon=159 \mathrm{M}^{-1} \mathrm{~cm}^{-1}$ which is measured for bromine vapors at $25^{\circ}$. For bromine water, Beylisset et. al. ${ }^{5}$ report an extinction coefficient $\epsilon=164 \mathrm{M}^{-1} \mathrm{~cm}^{-1}$ at a peak wavelength of $\lambda=392 \mathrm{~nm}$. When bromine is mixed with water, a hydrolysis reaction accompanied by a photo-chemical process takes place. ${ }^{4,8,9}$ This small loss of bromine could justify the lower value found for the extinction coefficient for aqueous solutions of bromine compared to bromine vapors values for the same wavelength $\left(\lambda=404 \mathrm{~nm}, \epsilon=159 \mathrm{M}^{-1} \mathrm{~cm}^{-1}\right)^{5}$ and for the peak wavelength that has shifted 
$\left(\lambda=415 \mathrm{~nm}, \epsilon=152 \mathrm{M}^{-1} \mathrm{~cm}^{-1}\right) \cdot{ }^{7}$
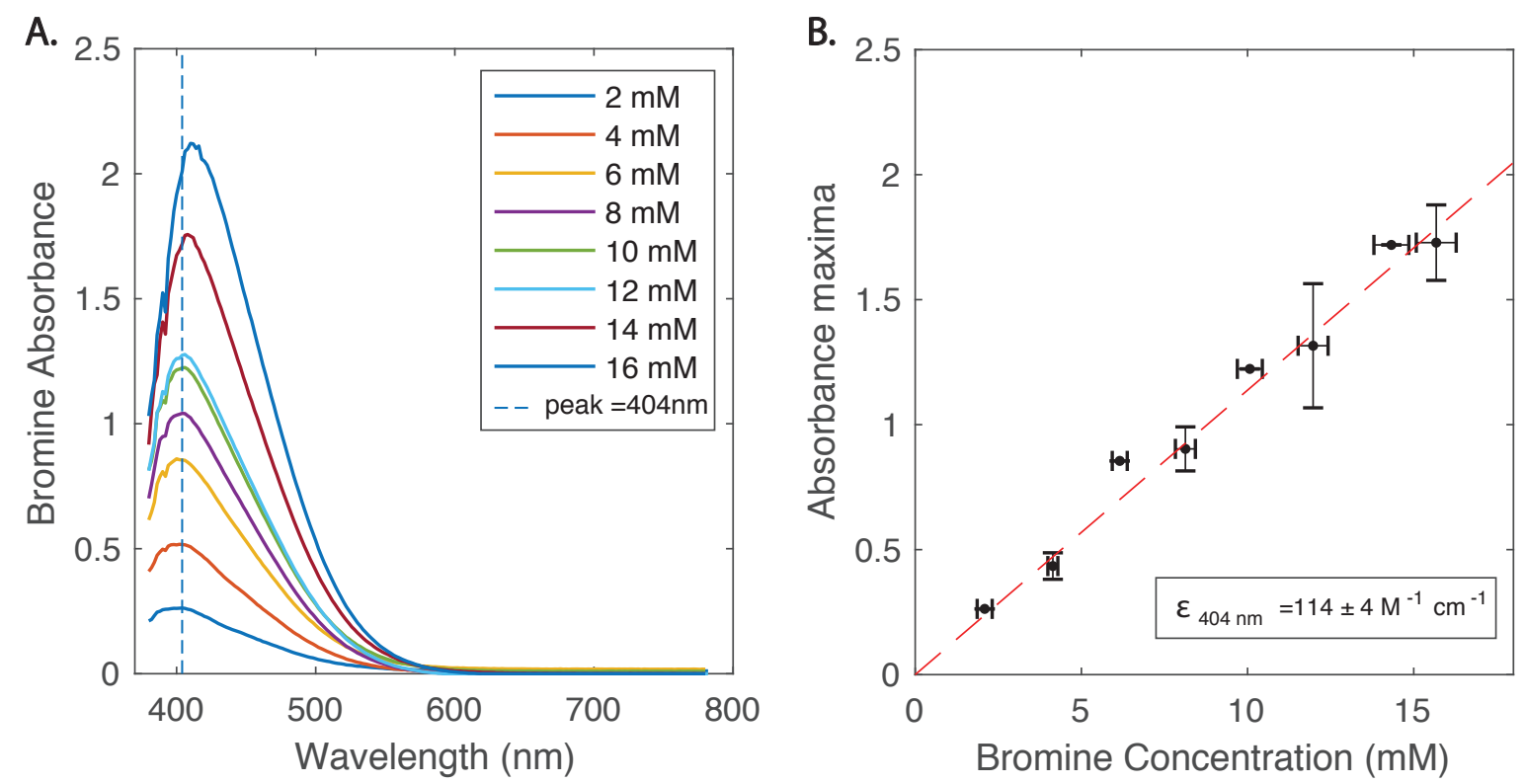

Figure S1: A. Absorption spectrum of brominated water as a function of bromine concentration. B. The absorbance maxima from (A) as a function of bromine concentration. The extinction coefficient at $\lambda=404 \mathrm{~nm}$ at room temperature; $\epsilon_{404 \mathrm{~nm}}=114 \pm 4 \mathrm{M}^{-1} \mathrm{~cm}^{-1}$.

A recommended way for minimizing the effect of the hydrolysis of bromine when mixed with water is to acidify the solution to $\mathrm{pH}=2$ with the addition of a $2 \mathrm{M}$ of sulfuric acid in the solution. ${ }^{4,8,9}$ Sulfuric acid is known to corrode the surface of the PDMS if the concentration is high ${ }^{10}$ which might affect the transport properties resulting in measuring different diffusion, reaction and partition coefficients. We did a short study using an acidic bromine water solution with sulfuric acid concentration of $80 \mathrm{mM}$, sodium bromate of $288 \mathrm{mM}$ and bromine $12 \mathrm{mM}$, which are the acidity conditions for the Belouzov-Zhabotinsky reaction. For more details see supplement section S12. The absorbance measurements were similar to those obtained with using bromine water of $12 \mathrm{mM}$ for both passivated and unpassivated PDMS. For this reason, we did not include sulfuric acid in our aqueous bromine solution. 


\section{Methods}

\section{S5. Experimental details}

All dilutions of the saturated bromine water were made by using $2 \mathrm{ml}$ Fisherbrand ${ }^{\mathrm{TM}}$ Disposable pasteur pipettes made from Borosilicate Glass mounted to a 200p Gilson pipette with an elastic plastic connecting tube of $6.80 \mathrm{~mm}$ inner diameter. The random error in the volume of saturated bromine water is $3.6 \%$. In the beginning of each experiment, we allowed the spectrometer to warm up for 5 minutes. Our spectrometer was calibrated by recording the absorption spectrum of the solvent, the deionized water, which serves as the blank. The absorbance noise is 0.04 . The bromine water concentration that we used in all our experiments is $12 \mathrm{mM}$, which is the same concentration that was also selected by A. A. Ben-Bassat. ${ }^{9}$ We obtained it by mixing $10 \mathrm{ml}$ of deionized water with $578 \pm 21 \mu \mathrm{l}$ of saturated bromine water.

For a complete set of data, we used two glass $10 \mathrm{~mm}$ cuvettes with glass stoppers (see Fig. S2). The first is referred to as the "reference" and the second as "measurement". The "measurement" cuvette contained a certain number of PDMS pieces. A volume of $2.5 \mathrm{ml}$ of $12 \mathrm{mM}$ bromine water was added to both cuvettes with the use of a Silicone Pipette Filler, 3-way valve connected with a $2 \mathrm{ml}$ Standard-Length Serological Pipette.

The absorption data were taken only from the aqueous phase of the two cuvettes. The measurement path length was kept constant (1 cm - length of the cuvette used). A small perforated layer of cyclic olefin copolymer (COC) was included in both cuvettes acting as a sieve and holding the PDMS pieces away from the optical measurement path. The COC

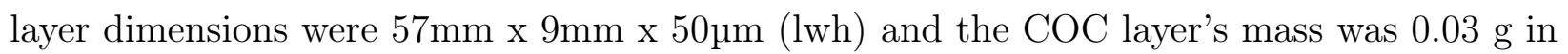
both cuvettes. Each COC film was perforated in 9 symmetrically placed locations in a $9 \mathrm{~mm}$ x $9 \mathrm{~mm}$ square in the center of the COC stripe. Each hole had a $1 \mathrm{~mm}$ diameter. To ensure that the concentration of bromine inside the cuvettes is homogeneous and not blocked by the COC layer, we generated several g's of acceleration by aggressively shaking by hand the 


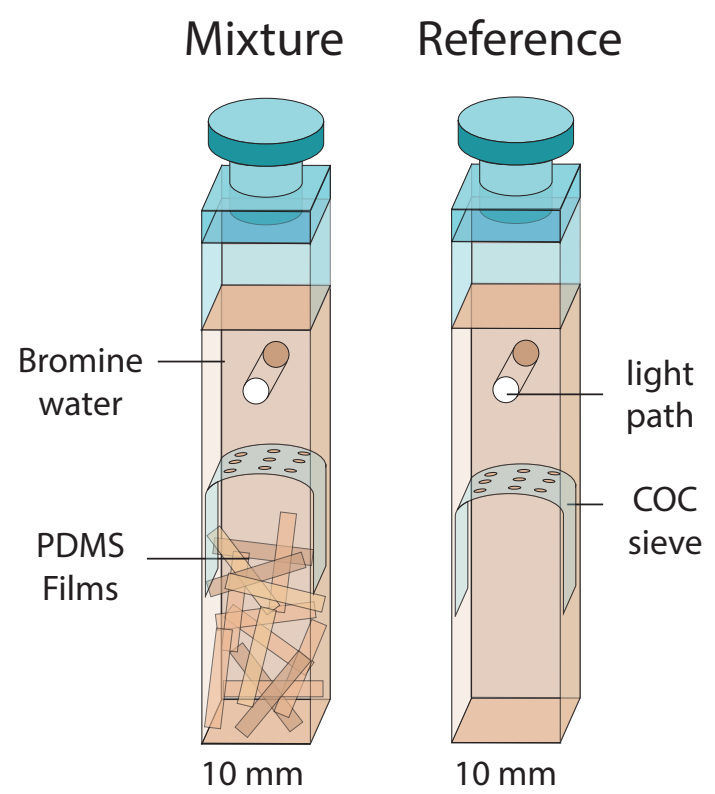

Figure S2: For every trial, two $10 \mathrm{~mm}$ cuvettes samples were studied: A cuvette used as reference containing only bromine water, and a second containing both PDMS films and bromine water. Initially, a $2.5 \mathrm{ml}$ bromine water was added to both cuvettes with the the same initial concentration $12 \mathrm{mM}$. A COC sieve was added to the two cuvettes with the same dimensions and mass.

cuvettes 5 - 10 times between each measurement. Any initial visible concentration gradient of bromine due to the COC disappears and the liquid appears uniform to the eye after one agitation. No other source of bromine consumption is expected as the absorbance of the reference cuvette remained constant during the course of the experiment for all trials.

The experiments on different PDMS masses causes the measuring path location to change position in the z- axis. A z-micrometer controlled stage was employed to adjust the height of the light path where the absorbance measurements were collected. The weighing error of the mass is $0.001 \mathrm{~g}$. The absorption collection data was averaged over one second using the software program Logger-Pro, which is compatible with the Vernier Ocean optics spectrometer used for this study. 


\section{Results and discussion}

\section{S6. Evidence of reaction: Qualitative evaluation of the PDMS trans- parency change}

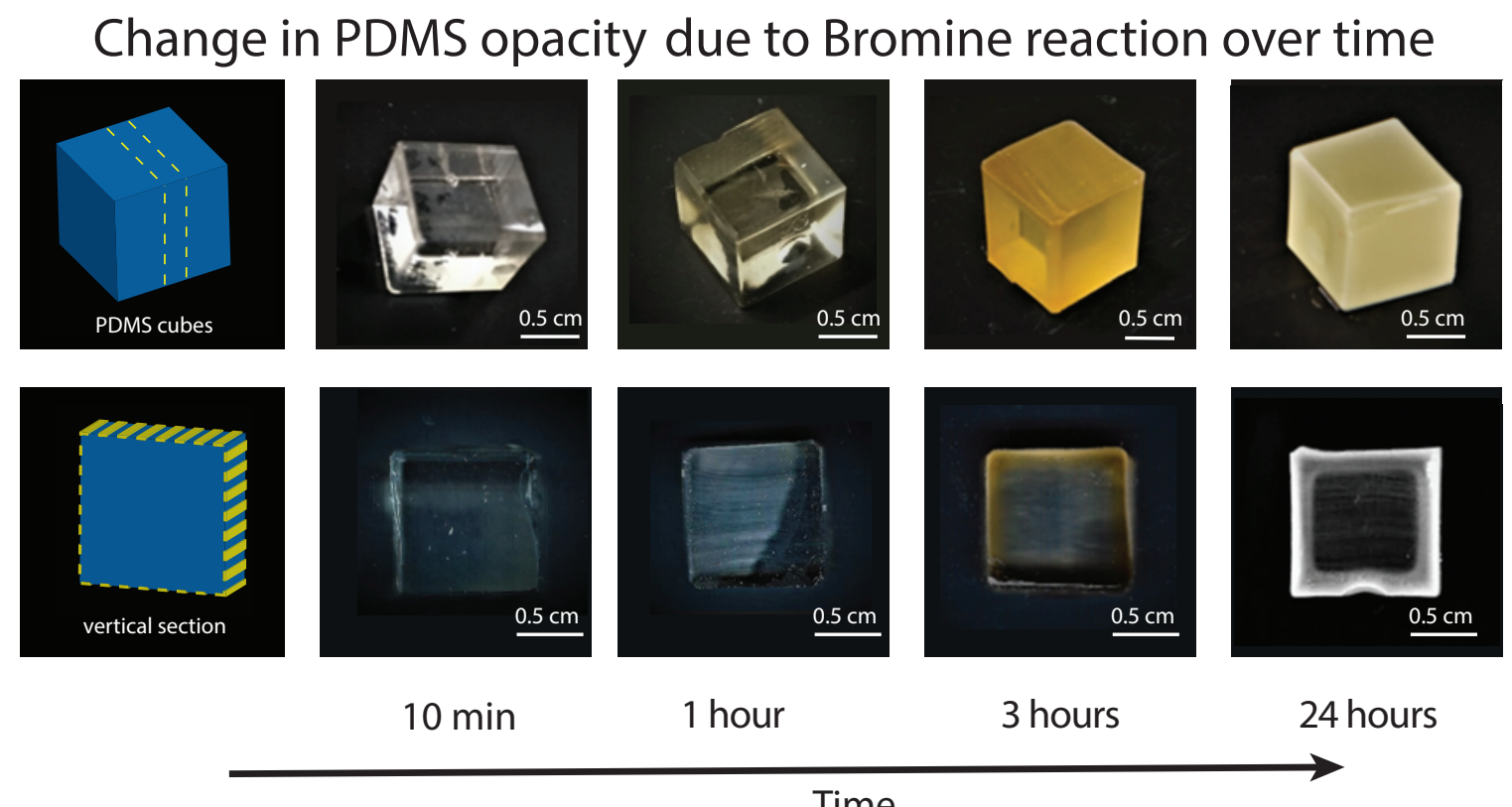

Figure S3: PDMS exposed to concentrated brominated water as a function of time. Top row: PDMS cubes of 1 cubic $\mathrm{cm}$. Bottom row: $2 \mathrm{~mm}$ thick slices from the top row. PDMS usually is a transparent. However, when it comes in contact with bromine-water, PDMS becomes less clear over time. This implies the presence of a reaction process in addition to a diffusion process. The degree of this non-transparency increases with time, a process that does not take place when PDMS is in contact with water for the same time period. No transparency or any other change was observed to the PDMS cube submerged to water for the same time period

Fig. S3 provides additional evidence for the existence of reaction and a change in transparency for PDMS with significant thickness. Four $1 \mathrm{~cm}$ x $1 \mathrm{~cm}$ x $1 \mathrm{~cm}$ (lwh) PDMS cubes were inserted into four glass beakers of $25 \mathrm{ml}$ and sealed with glass stoppers. A bromine water solution with concentration of $0.1 \mathrm{M}$ was inserted in each beaker at the same time. The solution was obtained by mixing $20 \mathrm{ml}$ of deionized water with $1.2 \mathrm{ml}$ of saturated bromine water. Each cube was removed and examined after a different time interval. The 
transparency of the PDMS cubes changed as a function of time. The PDMS became vividly stained by bromine after the first 3 hours but the cube lost this vivid color and appeared opaque after 24 hours. All bromine in the beaker was consumed. The examination of a vertically cut slice of each cube revealed that the surface changes with time. A non-transparent, white layer formed on the outside of the PDMS cubes after 24 hours.

\section{S7. Passivated PDMS thin films data}

Fig. S4, shows the absorbance vs time of passivated thin PDMS films as a function of mass. The data shown are the mean absorbance of 2 samples per PDMS mass for 8 trials in total. The data are fitted with Model $1 \mathrm{P}$ and Models $2 \mathrm{P}$ and the fitting results are tabulated and discussed in the main paper.

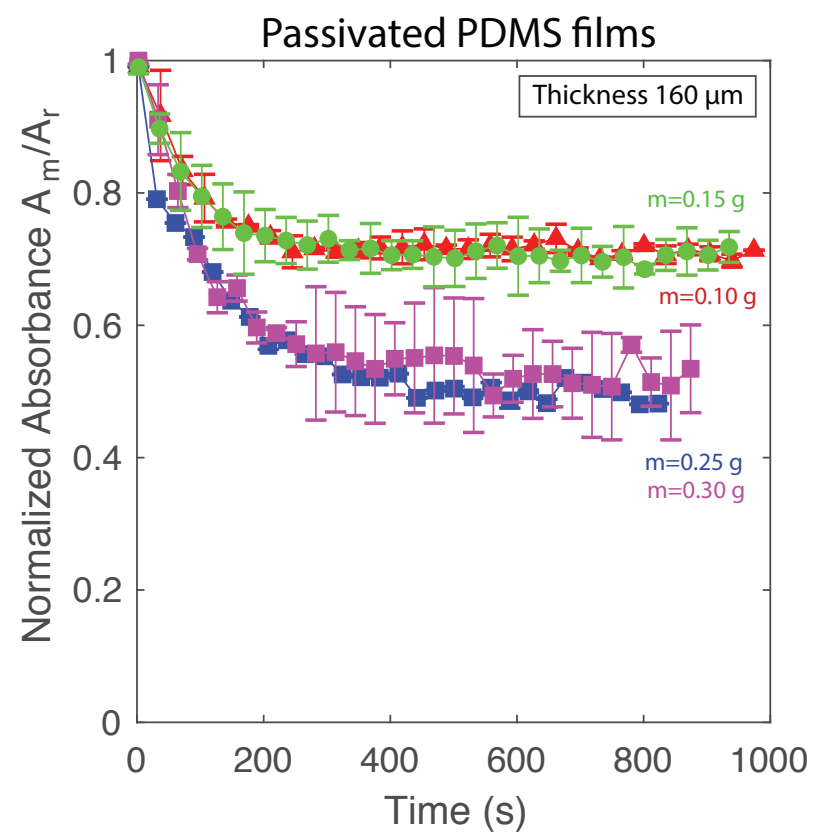

Figure S4: Sample of normalized absorbance data $\left(A=A_{m} / A_{r}\right)$ vs. time of thin, passivated PDMS films of constant thickness $(160 \pm 15 \mu \mathrm{m})$, as a function of mass. The initial concentration of bromine was $\left[\mathrm{Br}_{2}\right]=12 \mathrm{mM}$. Every $4^{\text {th }}$ data point is plotted. The data shown are the average of two samples for each PDMS weight. 


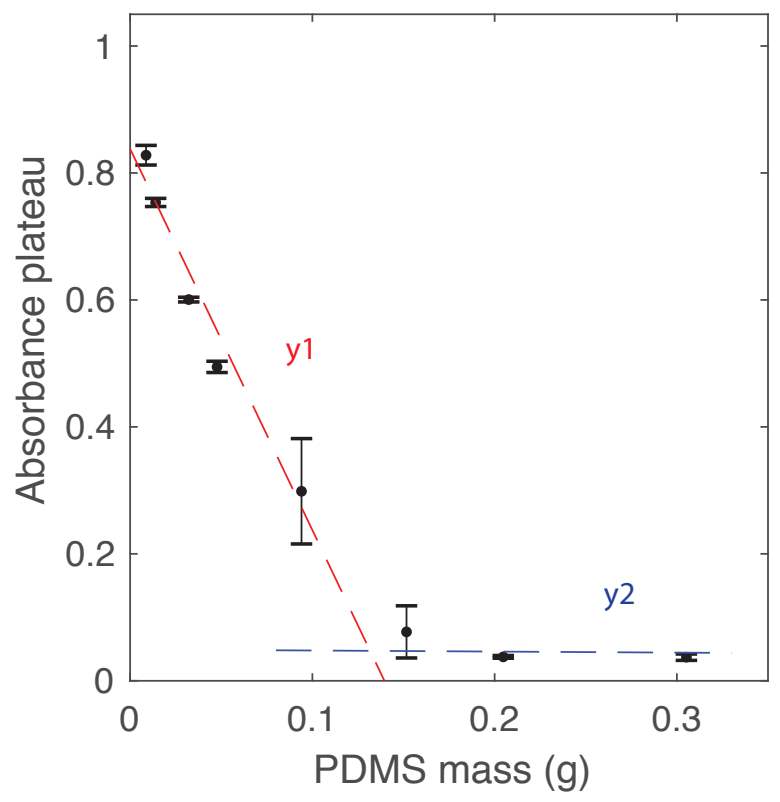

Figure S5: Absorbance plateau vs unpassivated PDMS mass for films of thickness $160 \mu \mathrm{m}$. The absorbance plateau corresponds to the mean of the last 10 normalized mixture absorbance measurements from the yellow shadowed region in Fig. 6 of the main paper, where the normalized absorbance of each sample has reached a time independent value. The initial concentration of bromine was $\left[\mathrm{Br}_{2}\right]=12 \mathrm{mM}$. Here we categorize the points into two groups. The low mass data linearly decreases with mass. The high mass data points are independent of mass. The two lines intersect at a mass of $0.12 \mathrm{~g}$. 


\section{S8. Estimation of the PDMS reactants concentration}

One of our fitting parameters is the concentration of the reactants in the PDMS volume. It is assumed that the reactants are uniformly distributed inside the PDMS volume. To increase the accuracy of our theoretical fitting models, we estimated the concentration of the reactants in PDMS using our unpassivated thin PDMS absorption data. Fig. S5 shows the mean of the last ten absorbance recorded values normalized with reference (yellow shadowed region in Fig. 6 of the main paper) as a function of PDMS mass. The data were separated into two groups that were fitted with two linear models. The absorbance for masses above $0.15 \mathrm{~g}$ are near zero. We neglect partitioning of bromine and consider that all the bromine in solutions has reacted with the PDMS. The two fitting lines were found to intersect for mass $m_{\text {critical }}=0.12 \mathrm{~g}$. This is the mass for which the moles of reactants in the PDMS are matched by the moles of bromine initially placed in the beaker.

By finding the volume of PDMS bulk using the mass $m_{\text {critical }}$ and PDMS density ( $\rho=$

$1.07 \mathrm{~g} / \mathrm{cm}^{3}$ ) and with the use of bromine concentration $\left[\mathrm{Br}_{2}\right]=12 \mathrm{mM}$ and bromine water volume $V_{\mathrm{Br}_{2}}=2.5 \mathrm{ml}$ ), we found the concentration of the reactants to be $w_{o}=244 \mathrm{mM}$ for PDMS. This value was selected as the initial value of the concentration of reactants fitting parameter $\left(w_{o}\right)$. However, the uncertainty to find this mass is higher toward lower masses, as there is an uncertainty in the slope of the line that fits the low mass data. Based on this uncertainty, in all our fits to Model $3 \mathrm{U}$ we restricted the fitting parameter $w_{o}$ to take values between 0 - $400 \mathrm{mM}$.

\section{S9. Acidic bromine solution}

PDMS is a transparent polymer. However, when it comes in contact with highly concentrated bromine water its transparency disappears. The degree of opacity is reduced by introducing sulfuric acid into the solution; the higher the concentration, the less opaque the PDMS becomes during Bromine exposure (see Fig. S6). The initial control sample was composed of a $1 \mathrm{~cm}^{3}$ PDMS cube (10:1 mixing ratio) in $5 \mathrm{ml}$ of DI water in a glass vial. All subsequent 
samples presented in Fig. S6 were composed of $1 \mathrm{~cm}^{3}$ PDMS cubes (10:1 mixing ratio) placed in a solution of $23 \mathrm{mM}$ bromine and $0-3 \mathrm{M}$ of sulfuric acid in glass vials. The vials were then sealed with a circular microscope coverglass inserted into the plastic cap to prevent bromine evaporation through the plastic. Photos were acquired of the PDMS in situ 3 hours after submerging the PDMS cubes in the brominated solutions.

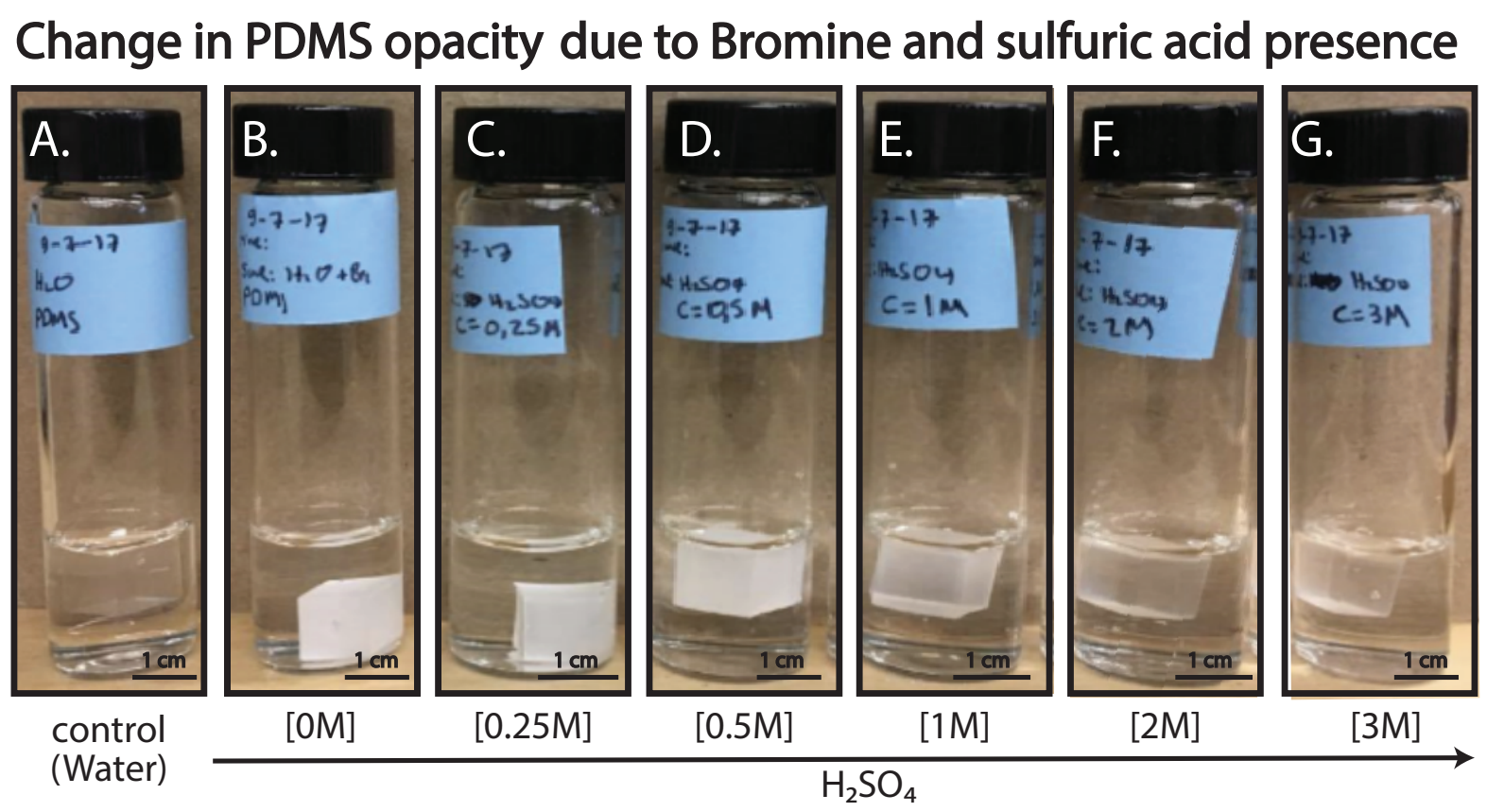

Figure S6: Images of PDMS in brominated water as a function of sulfuric acid concentration. All samples had initially the same initial concentration of bromine water $(23 \mathrm{mM})$ and all PDMS cubes had dimensions $1 \mathrm{~cm} \times 1 \mathrm{~cm} \times 1 \mathrm{~cm}$. The time the pictures recorded is 3 hours after incubation and the PDMS cubes are inside the original brominated solutions. A. The solution is $5 \mathrm{ml}$ bromine-free water and it serves as the control. B. The solution is bromine water $(23 \mathrm{mM})$ in contact with PDMS, which appears white-opaque.C-G The solutions (5ml) have increasing sulfuric acid concentrations. In each solution we added $23 \mathrm{mM}$ bromine. The PDMS opacity was observed to decrease as the concentration of the sulfuric acid increases. No Swelling of the PDMS was observed.

According to Givenchy et all, ${ }^{10}$ the surface of the PDMS becomes corroded when it comes in contact with a high concentrated sulfuric acid solution. When bromine is added to the acidic aqueous solution, other reactions may also take place due to the coexistence of sulfuric acid, bromine and PDMS. These reactions may reduce the amount of free bromine in the solution and cause changes to the surface properties of the PDMS which may affect 
the transport properties of bromine in PDMS. The four main chemical components of the Belousov-Zhabotinsky (BZ) reaction, apart from the two catalysts, that could are sodium bromide $(25 \mathrm{mM})$, sulfuric acid $(80 \mathrm{mM})$, sodium bromate $(288 \mathrm{mM})$ and malonic acid (400 mM).

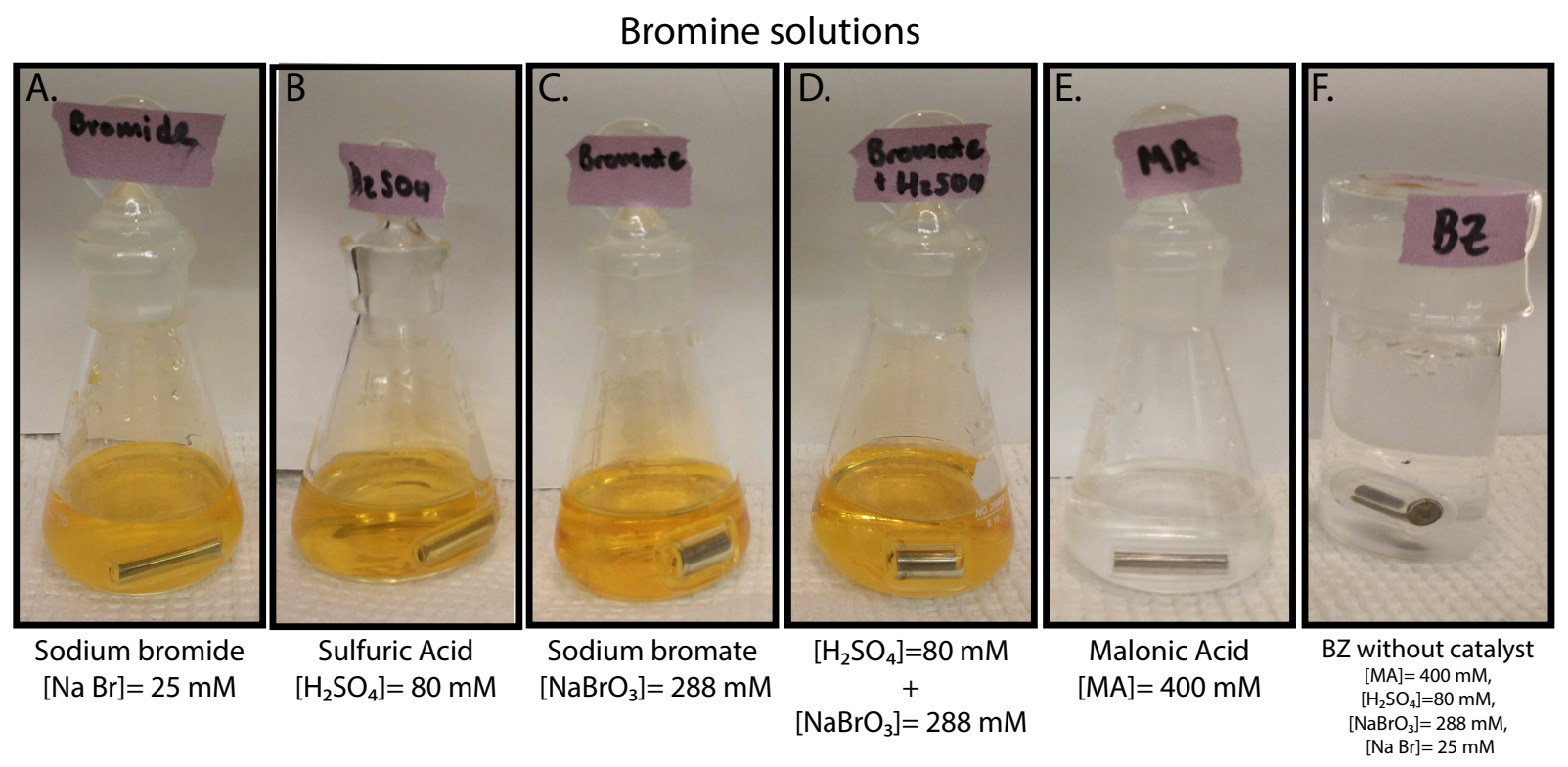

Figure S7: Images of Bromine water solutions. The initial concentration of bromine in solutions A- E is $1 \mathrm{mM}$. The bromine concentration of the solution $\mathbf{F}$ is $49 \mathrm{mM}$ and in this sample we also contain PDMS layers with mass $0.15 \mathrm{~g}$ and thickness $160 \mu \mathrm{m}$. The pictures were obtained 30 minutes after mixing and the solutions were well stirred with glass stir bars.

To identify if an aqueous bromine solution with the BZ reactants influence the bromine/PDMS reaction, we examined the stability of bromine in samples in the absence of PDMS, Fig. S7. We created four $10 \mathrm{ml}$ aqueous solutions of $12 \mathrm{mM}$ bromine and added to each one of the four chemicals used in the BZ reaction (Fig. S7A-D). By visually identifying the characteristic orange color of bromine, we concluded that bromine levels were stable in the sodium bromide, sulfuric acid, and sodium bromate solutions for at least one hour after being mixed (Fig. S7A-C). Similarly, we observed a stable bromine concentration in the presence of both sulfuric acid and sodium bromate (Fig. S7D). The orange color, however, completely disappears within seconds after mixing the same volume of saturated bromine water with $400 \mathrm{mM}$ of malonic acid (Fig. S7E), as expected due to the bromination of malonic acid. Fig. S7F 
shows that this is also the case when a $12 \mathrm{mM}$ bromine solution is mixed with all 4 components of the BZ reaction. More specifically, for this experiment we mixed a $20 \mathrm{ml}$ of solution adding initially $6 \mathrm{ml}$ of saturated bromine water and targeting a final aqueous bromine solution concentration of $49 \mathrm{mM}$. This is the same bromine concentration used for passivating the PDMS films. In this solution we also incubated $0.15 \mathrm{~g}$ of PDMS films with $160 \mu \mathrm{m}$ thickness and no change in transparency and color was observed after the addition of bromine water.
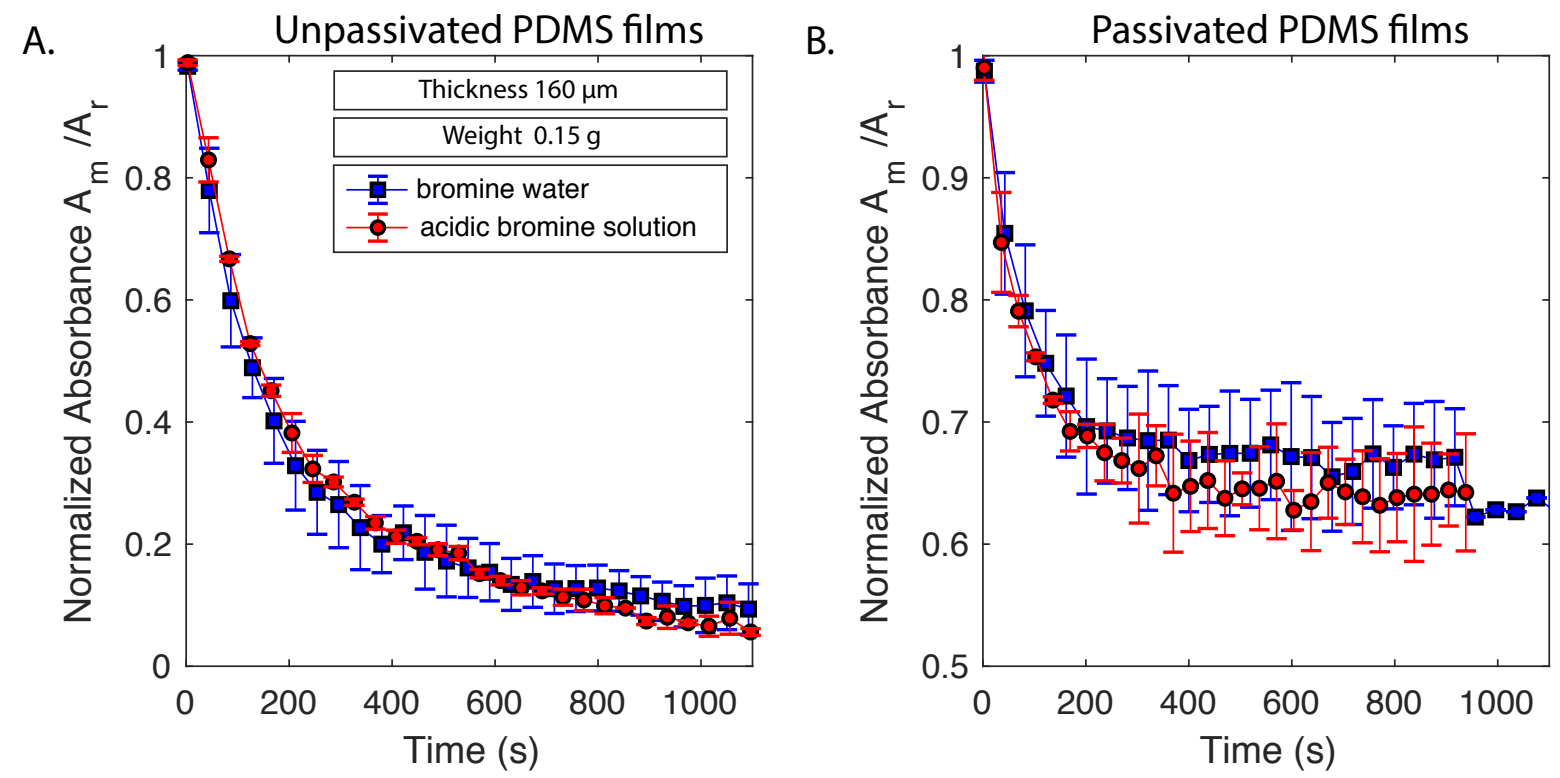

Figure S8: Absorbance of passivated and unpassivated PDMS films in contact with acidic bromine solution ( $80 \mathrm{mM}$ sulfuric acid, $288 \mathrm{mM}$ sodium bromate). All PDMS layers used for acquiring the data shown in both panels $\mathrm{A}$ and $\mathrm{B}$ have $160 \mu \mathrm{m}$ thickness and $0.15 \mathrm{~g}$ mass. The data shown are the average of two samples acquired with the use of the acidic bromine solution $12 \mathrm{mM}$ (red dots) and three acquired with the use of bromine water $12 \mathrm{mM}$ (blue dots). Every $4^{\text {th }}$ data point is plotted. A. Unpassivated PDMS absorbance as a function of time. B. Passivated PDMS absorbance as a function of time.

In order to identify if the sulfuric acid concentration in the BZ reaction may result to different coefficient values, we recorded data using the same experimental protocol as described in supplementary section S5 using passivated and unpassivated PDMS films in two different solutions (Figure S8). The first solution, which served as our control, was bromine water with bromine concentration $12 \mathrm{mM}$. The second was an acidic bromine aqueous solution containing $80 \mathrm{mM}$ sulfuric acid, $288 \mathrm{mM}$ sodium bromate, and $12 \mathrm{mM}$ bromine. This 
solution was selected because it contains the acids of the oscillatory BZ reaction typically used in PDMS network experiments. Note that these solutions do not include malonic acid, which bromine readily brominates.

Table S7: Summary of fitting results for Model 1P and Model 2P. Passivated PDMS films with constant mass $(\mathrm{m}=0.15 \mathrm{~g})$ incubated in bromine water and acidic bromine solution ( $80 \mathrm{mM}$ sulfuric acid, $288 \mathrm{mM}$ sodium bromate) with bromine concentration $12 \mathrm{mM}$. The absorbance data is in Fig. S8A

\begin{tabular}{lll}
\hline Model 1P & & \\
\hline Coefficients & Bromine water & Acidic bromine solution \\
\hline Partition Coefficient $\left(P_{1}\right)$ & $12 \pm 2$ & $9 \pm 2$ \\
Diffusion Coefficient $D_{u}\left(10^{-9} \mathrm{~m}^{2} / \mathrm{s}\right)$ & $0.022 \pm 0.0049$ & $0.021 \pm 0.0028$ \\
\hline \hline Model 2P & & \\
\hline Coefficients & Bromine water & Acidic bromine solution \\
\hline$k_{-}\left(\mathrm{s}^{-1}\right)$ & $0.0077 \pm 0.0022$ & $0.0062 \pm 0.00092$ \\
$k_{+}\left(\mathrm{s}^{-1}\right)$ & $0.082 \pm 0.024$ & $0.054 \pm 0.013$ \\
Partition Coefficient $\left(P_{2}^{*}\right)$ & $12 \pm 2$ & $10 \pm 1$ \\
\hline
\end{tabular}

Table S8: Fitting parameters for Model 3U for unpassivated PDMS films. The parameters that are varied are designated by "fit". Not all the parameters are independent as $P_{2}^{*}=$ $k_{+} / k_{-}$. Model $3 \mathrm{U}$ accounts for reversible binding of bromine to PDMS, free diffusion of bromine in PDMS and irreversible reaction between free bromine and PDMS.

\begin{tabular}{lll}
\hline Model 3U & & \\
\hline Parameters & Bromine water & Acidic bromine solution \\
$P_{2}^{*}$ & 12 & 10 \\
$k_{+}[1 / \mathrm{s}]$ & 0.082 & 0.054 \\
$k_{-}[1 / \mathrm{s}]$ & 0.0077 & 0.0062 \\
$D_{u}\left[10^{-9} \mathrm{~m}^{2} / \mathrm{s}\right]$ & fit & fit \\
$k_{u w}$ & fit & \\
$w_{0}[\mathrm{mM}]$ & fit $[0-400]$ & fit $[0-400]$ \\
$t_{\text {shift }}$ & fit & fit \\
\hline
\end{tabular}

Figure S8A and Figure S8B compare the decay absorbance trends of unpassivated and passivated PDMS films in the presence and absence of acid. All PDMS samples had the same mass $\mathrm{m}=0.15 \mathrm{~g}$ and the same thickness $160 \mu \mathrm{m}$. We eliminated any effect of the heat of mixing of sulfuric acid by mixing solutions inside a bucket of ice. The produced 
Table S9: Summary of fitting results for unpassivated PDMS films with thickness $160 \mu \mathrm{m}$ and with mass $\mathrm{m}=0.15 \mathrm{~g}$ incubated in bromine water and acidic bromine solution with bromine concentration $12 \mathrm{mM}$. The absorbance data is in Fig. S8B

\begin{tabular}{lll}
\hline Model 3U & & \\
\hline Coefficients & Bromine water & Acidic bromine solution \\
\hline Reaction constant $k_{u w}\left[10^{-4} \mathrm{~s}^{-1} \mathrm{mM}^{-1}\right]$ & $8.31 \pm 2.58$ & $7.17 \pm 0.95$ \\
Concentration of reactants $\left(w_{o}[\mathrm{mM}]\right)$ & $232 \pm 19$ & $265 \pm 1$ \\
Diffusion constant $D\left[10^{-9} \mathrm{~m}^{2} / \mathrm{s}\right]$ & $0.40 \pm 0.11$ & $0.45 \pm 0.067$ \\
\hline Bromine reaction rate $\left(k_{\mathrm{Br}}[1 / \mathrm{s}]\right)$ & $0.19 \pm 0.057$ & $0.19 \pm 0.025$ \\
Reaction Diffusion Length $(\mathrm{L}[\mathrm{\mu m}])$ & $45 \pm 2$ & $49 \pm 0.27$ \\
\hline
\end{tabular}

stock solutions were left to reach room temperature at least 24 hours before using them in any experiment. When sulfuric acid solutions were mixed with aqueous bromine and the PDMS films inside the cuvettes, no rise in temperature was observed. Partition, reaction and diffusion coefficients were estimated and compared by fitting the passivated PDMS films data to Model 1P and 2P (see table S7) and the unpassivated PDMS films data to Model 3U (see table S8 and table S9). In both cases, the absorbance vs time are identical. This proves that sulfuric acid and sodium bromate in concentrations employed in oscillatory BZ experiments in PDMS networks do not affect the interaction of bromine and PDMS.

\section{Modeling details}

\section{S10. Effective Diffusivity of Model 3U}

In the absence of irreversible reactions and in the limit of fast reaction kinetics, we now show that temporary immobilization of bromine through binding to the PDMS reduces the effective diffusivity of bromine. In order to obtain an analytical result, we will make assumptions in this analysis that are not valid for the experiments. However,the result will qualitatively demonstrate why reversible binding reduces the apparent diffusion. To do so we begin with the simplified model $3 \mathrm{U}$ by setting $k_{u w}=0$, which gives 


$$
\frac{\partial u}{\partial t}=D_{u} \frac{\partial^{2} u}{\partial x^{2}}-k_{+} u+k_{-} v
$$

and

$$
\frac{\partial v}{\partial t}=k_{+} u-k_{-} v
$$

We Laplace transform the unknown functions in time only such that $L\{u(x, t)\} \rightarrow U(x ; s)$ and $L\{v(x, t)\} \rightarrow V(x ; s)$, yielding

$$
s U=D_{u} \frac{d^{2} U}{d x^{2}}+k_{-}\left(V-P_{2} U\right)
$$

and

$$
s V=k_{-}\left(P_{2} U-V\right)
$$

Where, without any loss of generality, we've let $u(x, t)=0$ and $v(x, t)=0$. Solving for $V$ in Eqn. 4 gives

$$
V=\frac{k_{-} P_{2} U}{k_{-}+s}
$$

and substituting into 3 and rearranging gives

$$
0=D_{u} \frac{d^{2} U}{d x^{2}}+U\left(\frac{-P_{2} s-s-\frac{s^{2}}{k_{-}}}{1+\frac{s}{k_{-}}}\right)
$$

In the limit of fast kinetics, $k_{-} \rightarrow \infty$, dynamics simplify to the algebraic equation $V=P_{2} U$ and modified diffusion equation

$$
U s=D_{u}^{*} \frac{d^{2} U}{d x^{2}}
$$

where $D_{u}^{*}=\frac{D_{u}}{P_{2}+1}$ is the effective, reduced diffusivity of Bromine within PDMS. Consequently, any physical value $\left(P_{2}>0\right)$ reduces mass transport. 


\section{S11. Analytical Solution of Model 2P}

We repeat Model 2P (eqn. 6 in the main text) here for convenience

$$
\begin{aligned}
& \frac{d u}{d t}=\phi\left(k_{-} v-k_{+} u\right) \\
& \frac{d v}{d t}=\left(k_{+} u-k_{-} v\right) .
\end{aligned}
$$

Integrating the 2 nd order system gives

$$
\begin{aligned}
& u(t)=\frac{u_{1}}{k}\left(k_{-}+\phi k_{+} e^{-k t}\right), \\
& v(t)=\frac{u_{1} k_{+}}{k}\left(e^{-k t}-1\right),
\end{aligned}
$$

where $k=k_{-}+k_{+} \phi$, and $u_{1}=(1-\phi) u_{\infty, 0}$ is the adjusted initial condition to account for the (unmodeled) rapid diffusion that occurs before the reversible reactions begin.

\section{Movies}

Movie S1: Passivated PDMS films color change after incubation

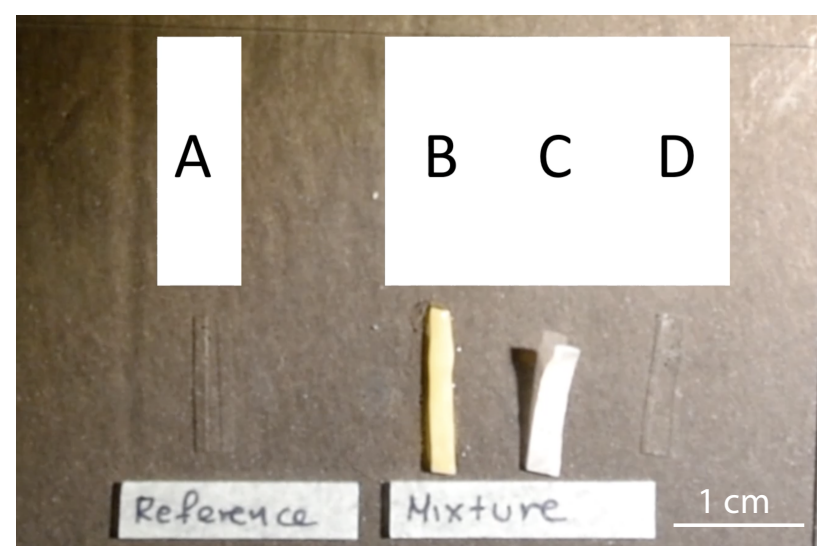

Figure S9: Temporal evolution of thin PDMS films after immersion in brominated water)

Evolution of the color change observed after immersion of thin passivated PDMS films in 
brominated water, $26.7 \mathrm{mM}$, for 46 hours. The thickness of the films is (160 $\pm 15 \mu \mathrm{m})$. Four PDMS sample layers are presented: The reference $(\mathrm{A})$ is an unpassivated PDMS transparent elastomer layer immersed in water for 46 hours. The passivated PDMS film (B) shows the color change due to bromine presence immediately after removal. Initially it appears opaque and orange and it gradually looses its color within the first 2 minutes exposed in air in room temperature and becomes white and opaque. The passivated PDMS film after 10 minutes in contact with room air (C) remains white and opaque. The passivated PDMS film after 2 hours (D) in contact with room air has recovered its transparency.

\section{Movie S2: Passivated PDMS films transparency restoration}

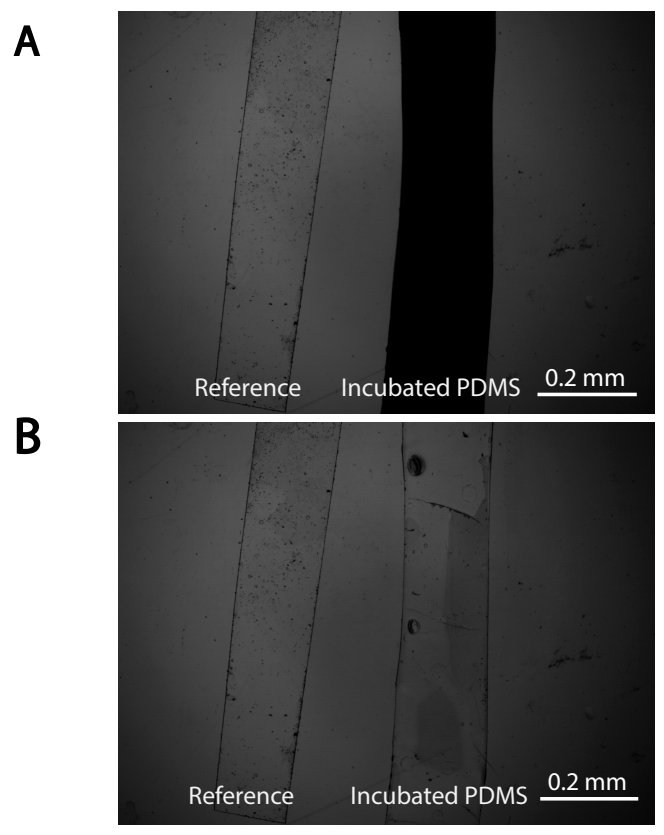

Figure S10: Passivated PDMS films transparency restoration. A.Initial transparency state. B.Final transparency state.

The transparency restoration of passivated PDMS films. The films were immersed for 46 hours in $26.7 \mathrm{mM}$ brominated water. Then they removed and left in the fume hood for 10 minutes until they lost their orange color and they appear white and opaque. The video starts recording 10 minutes after its removal from the bromine water. The PDMS film's 
transparency recovers after approximately 3 hours. A PDMS film (reference), incubated inside water for 46 hours and serves as a control also appears to the left.

\section{References}

(1) Mackison, R. S., F. W.and Stricoff; Partridge, J. J. e. NIOSH/OSHA - Occupational Health Guidelines for Chemical Hazards. Washington, DC: U.S. Government Printing Office Jan. 1981, 3 VOLS.

(2) Hocheng, H.; Chen, C.-M.; Chou, Y.-C.; Lin, C.-H. Study of novel electrical routing and integrated packaging on bio-compatible flexible substrates. Microsystem Technologies 2010, 16, 423-430.

(3) Goldschleger, I. U.; Senekerimyan, V.; Krage, M. S.; Seferyan, H.; Janda, K. C.; Apkarian, V. A. Quenched by ice: Transient grating measurements of vibronic dynamics in bromine-doped ice. The Journal of Chemical Physics 2006, 124, 204507.

(4) Gutmann, H.; Lewin, M.; Perlmutter-Hayman, B. Ultraviolet absorption spectra of chlorine, bromine, and bromine chloride in aqueous solution. The Journal of Physical Chemistry 1968, 72, 3671-3673.

(5) Bayliss, N.; Cole, A.; BG, G. The Visible Absorption Spectrum of Bromine in Solution. Australian Journal of Chemistry 1948, 1, 472-479.

(6) Acton, A. P.; Aickin, R. G.; Bayliss, N. S. The Continuous Absorption Spectrum of Bromine: A New Interpretation. The Journal of Chemical Physics 1936, 4, 474-479.

(7) Bayliss, N. S.; Rees, A. L. G. Effect of Foregin Gases on the Continuous Absorption Spectrum of Bromine. Nature 1939, 143, 560.

(8) Child, C. L.; Walker, O. J. The continuous absorption spectrum of bromine in solution. Transactions of the Faraday Society 1938, 34, 1506-1515. 
(9) Ben-Bassat, A. A. Studies on the Absorption Spectra of Aqueous Bromine, Bromine- $\mathrm{NaCl}$ and Bromine- NaBr. Israel Journal of Chemistry 1973, 11, 781-789.

(10) de Givenchy, E. T.; Amigoni, S.; Martin, C.; Andrada, G.; Caillier, L.; Géribaldi, S.; Guittard, F. Fabrication of superhydrophobic PDMS surfaces by combining acidic treatment and perfluorinated monolayers. Langmuir 2009, 25, 6448-6453. 